\title{
A new face in the line of defense against dementia
}

I In 1984 an act of Congress directed the NIH, specifically the National Institute on Aging, to establish a number of Alzheimer's Disease Research Centers (ADRCs) that would provide the infrastructure to support and advance research on the disease throughout the United States. Each center would be funded, currently at a total of \$6.5 million, for a period of 5 years, after which it would come up for competitive renewal. This year the University of California, San Francisco (UCSF) Memory and Aging Center became the newest ADRC and was the only new institution to be awarded this highly competitive grant in the 2004 funding period. (For the complete list of centers, see http:// www.alzheimers.org/adcdir.htm\#tex.)

Being named an ADRC brings special advantages to a research institute, because it becomes part of a larger interactive network and takes on a leadership role for the dementia research community. Bruce Miller, the director of the UCSF Memory and Aging Center and principal investigator of the new ADRC, explained what the designation would mean for their community of researchers. "I think that the idea of doing things collectively is what's so exciting about these centers," he told the JCI. "You become part of an Alzheimer's center network ... a national group that thinks collectively about these disorders. You participate in the national trials with new and exciting medication. You share certain aspects of the database." The database resources include material from a national genetic bank, which allows centers access to information on rare families that do not have the usual phenotypic or genotypic characteristics of families with dementia. Tissues, DNA samples, and pathology samples are also available to members of the network.

Miller felt there were several reasons why the UCSF center was chosen to be an ADRC. He noted that the exceptional basic research that goes on at the university certainly contributed but also thought that another important element was the diversity of its patient populations, especially since this center will be one of the few that will focus not just on Alzheimer disease, but also on other forms of dementia.

"We see a variety of degenerative dementias," said Miller, "and see many patients with disorders that are considered somewhat unusual, such as the frontotemporal dementias and the prion-related diseases. We do pretty

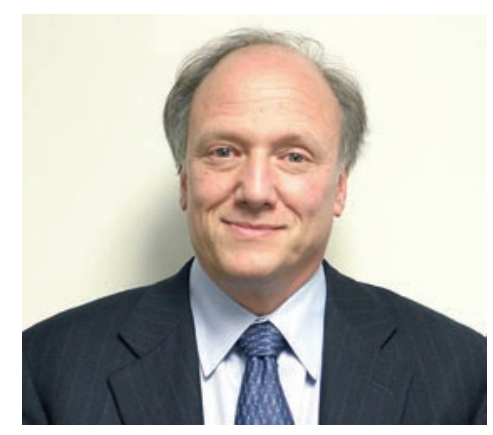

Bruce Miller looks forward to helping to organize the national effort against dementias.

extensive phenotyping on these populations - more than just simple and traditional neuropsychological testing. [For example, we do] very comprehensive imaging, very extensive data collection on personality, emotion, and other things that we think may be the earliest manifestations of degenerative diseases."

Miller laid out the main goals for their center, which include promoting further integration of data collected by researchers and clinicians at 3 institutes: the UCSF Memory and Aging Center, the San Francisco Veterans Affairs Medical Center, and the UCSF-affiliated Gladstone Institute of Neurological Disease.

Miller said that their work will concentrate on "the careful characterization of the earliest stages of both Alzheimer's and nonAlzheimer's degenerative dementias. So, we are not focused only on amnesia, but on language disorders [and] emotional/behavioral disorders in the earliest stages of the illness. That requires us to use different tools. [Currently] there is a lot of focus on the hippocampus and memory, and I think to diagnose Alzheimer's disease, where the disease doesn't begin in the hippocampus, you need different imaging strategies, different cognitive strategies. We think a lot about the frontal lobes, what the frontal lobes do, and how to capture deficits in the early stages when these start to break down. Similarly, we think a lot about the amygdala anterior temporal lobe, where other cases of frontotemporal dementia begin. This has forced us to think about language in a different way than the other traditional approaches to Alzheimer's disease, and emotion and personality, too."

Five main research cores have been established to achieve these research objectives, each with its own special tools, directives, and funds for specific projects from this NIH grant. The Clinical Core, which allows doctors to develop new diagnostic strategies, will now handle a greater number of patients. Miller noted that for this, "we are developing better probes of emotion, personality, and language, better ways of capturing change."

The team at the Imaging Core, directed by Michael Weiner, a UCSF professor of radiology at the SF Veterans Affairs Medical Center, will see many of the patients from the Clinical Core and will be developing their state-of-the-art 4.0 Tesla magnet for magnetic resonance imaging (MRI) as a tool for understanding dementia.

The Neuropathology Core, which is directed by Steven DeArmond, a UCSF professor of pathology and neuropathology, will be analyzing tissue samples from brain biopsies and autopsies from the ADRCs shared resources to study specific forms of dementia, including the non-Alzheimer forms.

All data that is obtained throughout the center will be amassed and disseminated throughout the ADRC network by the Data Management and Biostatistical Core. The UCSF ADRC also has an Administration, Education, and Outreach Core that will work to educate and to increase services for commonly underserved populations of patients, in particular Asian-Pacific Islanders.

Miller also highlighted that the funding provides the means to enhance UCSF's already high-quality basic research. "We are looking at different molecules than some of the other centers," he explained. "Lennart Mucke is thinking about a protein called calbindin, which he thinks may be the first protein to go awry in Alzheimer's disease. And Stan Prusiner thinks about prions. For some of our pilot projects, the young scientists focus on Tau, which is another protein that has received a little less attention at some of the other centers."

With the mean age in the US continuing to climb, and therefore the burden of dementia diseases on the country rising, the rapid translation of current research advances into diagnostics, treatments, and cures becomes increasingly important. This newest of the ADRCs, though it has the talent and tools to enhance the ADRC network in deciphering dementia, still faces a formidable foe indeed.

\section{Laurie Goodman}

